Research Article

\title{
On the Graph of Solution Mappings of Variational Inequalities Problems
}

\author{
Qi-Qing Song \\ College of Science, Guilin University of Technology, Guilin 541004, China \\ Correspondence should be addressed to Qi-Qing Song; songqiqing@gmail.com
}

Received 9 December 2013; Revised 27 March 2014; Accepted 27 March 2014; Published 9 April 2014

Academic Editor: Yuming Xing

Copyright (C) 2014 Qi-Qing Song. This is an open access article distributed under the Creative Commons Attribution License, which permits unrestricted use, distribution, and reproduction in any medium, provided the original work is properly cited.

This paper shows the property of homeomorphism between the space of monotone variational inequalities problems and the graph of their solution mappings.

\section{Introduction and Preliminaries}

It is well known that variational inequalities were introduced by Hartman and Stampacchia in the 1960s; see [1, 2]. Variational inequalities have become important methods for the study of many problems such as optimization problems, nonlinear complementary problems, fixed-point problems, saddle-point problems, and Nash equilibrium problems; see $[3,4]$ and references therein.

Let $K$ be compact convex subset of $\mathbb{R}^{n}$ and $f: K \rightarrow \mathbb{R}^{n}$ a mapping. A classic variational inequality problem is to find a point $\bar{x} \in K$ such that

$$
(y-\bar{x})^{T} f(\bar{x}) \geq 0, \quad \forall y \in K .
$$

A basic characteristic of monotone variational inequalities is the clarity of the construction of their solution sets, which attracts much attention in theoretical studies. Monotone variational inequalities are also important aspects in algorithm designs. We intend to recall some notions with monotonicity.

Definition 1. A function $f: K \rightarrow \mathbb{R}^{n}$ is said to be monotone if $(f(x)-f(y))^{T}(x-y) \geq 0$, for all $x, y \in K$; $f$ is said to be strictly monotone if $f$ is monotone, and if $(f(x)-f(y))^{T}(x-$ $y)=0$, then $x=y ; f$ is strongly monotone if there exists a constant $c>0$ such that $(f(x)-f(y))^{T}(x-y) \geq c\|x-y\|^{2}$, for all $x, y \in K$.
Denote by $M$ the set as follows:

$M=\left\{f: f: K \longrightarrow \mathbb{R}^{n}\right.$ is continuous and monotone $\}$.

For any two $f_{1}, f_{2} \in M$, we adopt the uniform metric $\rho\left(f_{1}, f_{2}\right)$ between $f_{1}$ and $f_{2}$; that is,

$$
\rho\left(f_{1}, f_{2}\right)=\max _{x \in K}\left\|f_{1}(x)-f_{2}(x)\right\| .
$$

We write $\rho\left(f_{n}, f_{0}\right) \rightarrow 0$ as $f_{n} \stackrel{\rho}{\rightarrow} f_{0}$. For each $f \in M$, if a point $\bar{x}$ is a solution of the problem (1) with respect to $f$, we denote it as $\bar{x} \in V(f)$. Then, we define a set-valued mapping $V$ from $M$ to $K$. For each $f \in M$, by the finite dimensional case of Theorem 1.4 in Chapter III in [5], it holds that $V(f) \neq \emptyset$. In fact, $V(f)$ is a closed convex set for each $f \in M$, and if $f$ is strictly monotone, $V(f)$ is a singleton set.

Let $N$ be the graph of the set-valued mapping $V$; that is,

$$
N=\{(f, x) \in M \times K \mid x \in V(f)\} .
$$

Noting the uniqueness of strictly monotone variational inequalities, the graphs $N$ and $M$ may show some relations, and this paper aims to illustrate the construction between variational inequalities problems and the graph of solution mappings in detail. The homeomorphism between $M$ and $N$ is revealed, which is linked closely with the stability of solutions of variational inequalities (1).

For each $\left(f, x^{*}\right) \in N$, let $\phi\left(f, x^{*}\right)=f-C_{x^{*}}$, where $C_{a}$ denotes the constant mapping such that $C_{a}(x)=a$, for all $x \in K$. Clearly, $C_{x^{*}}$ is monotone; hence, $C_{x^{*}} \in M$. Then, $\phi$ is a mapping from $N$ to $M$. 


\section{Properties of the Graph Space of Solution Mappings}

Theorem 2. The spaces $N$ and $M$ are homeomorphic.

Proof. For each $f \in M$, let $\psi(f)=\left(f+C_{\bar{x}}, \bar{x}\right)$, where $\bar{x}$ is the unique point in $V(f+I)$ ( $I$ is the identity mapping on $K)$; then, $\psi$ is a mapping from $M$ to $M \times K$. We need to show the following five steps.

(a) $\psi$ maps $M$ onto $N$. For each $f \in M$, since $\bar{x} \in V(f+$ $I)$, we have $(y-\bar{x})^{T}(f(\bar{x})+\bar{x}) \geq 0$, for all $y \in K$; that is, $\bar{x} \in V\left(f+C_{\bar{x}}\right)$.

(b) $\psi$ is continuous on $M$. In order to show this, firstly, we prove that $N$ is closed. Let $\left(g_{n}, z_{n}\right) \in N(n=1,2, \ldots)$ and $\left(g_{n}, z_{n}\right) \rightarrow\left(g_{0}, z_{0}\right) \in M \times K\left(g_{n} \stackrel{\rho}{\rightarrow} g_{0}\right.$ and $\left.\left\|z_{n}-z_{0}\right\| \rightarrow 0\right)$. Then, $z_{n} \in V\left(g_{n}\right)$; that is,

$$
\left(y-z_{n}\right)^{T} g_{n}\left(z_{n}\right) \geq 0, \quad \forall y \in K .
$$

As $n$ is close to infinity for (5), noting that $g_{0}$ is continuous, we have

$$
\left(y-z_{0}\right)^{T} g_{0}\left(z_{0}\right) \geq 0, \quad \forall y \in K .
$$

Then, $z_{0} \in V\left(g_{0}\right)$. Consequently, $N$ is closed. Hence, the setvalued mapping $V$ is upper semicontinuous on $M$.

Next, let $f_{n} \in M(n=1,2, \ldots)$ and $f_{n} \rightarrow f_{0} \in M$. Then, for each $n=0,1,2, \ldots, \psi\left(f_{n}\right)=\left(f_{n}+C_{x_{n}}, x_{n}\right)$, where $x_{n}$ is the unique point in $V\left(f_{n}+I\right)$. We need to show that $\left(f_{n}+\right.$ $\left.C_{x_{n}}, x_{n}\right) \rightarrow\left(f_{0}+C_{x_{0}}, x_{0}\right)$. Since $V$ is upper semicontinuous on $M$, for any open set $U \supset V\left(f_{0}+I\right)=\left\{x_{0}\right\}$, there exists a number $m$ such that, for each $n>m, V\left(f_{n}+I\right)=\left\{x_{n}\right\} \subset U$. Therefore, we can obtain that $x_{n} \rightarrow x_{0}$. For each $x \in K$, as $n$ gets close to infinity, since the right side of the following inequality,

$$
\begin{aligned}
& \left\|f_{n}(x)+x_{n}-\left(f_{0}(x)+x_{0}\right)\right\| \\
& \quad \leq\left\|f_{n}(x)-f_{0}(x)\right\|+\left\|x_{n}-x_{0}\right\|,
\end{aligned}
$$

tends to zero, we have $\left(f_{n}+C_{x_{n}}\right) \stackrel{\rho}{\rightarrow}\left(f_{0}+C_{x_{0}}\right)$.

(c) $\phi$ is continuous on $N$. Let $\left(f_{n}, x_{n}\right) \in N(n=1,2, \ldots)$ and $\left(f_{n}, x_{n}\right) \rightarrow\left(f_{0}, x_{0}\right) \in N\left(f_{n} \stackrel{\rho}{\rightarrow} f_{0}\right.$ and $\| x_{n}-$ $\left.x_{0} \| \rightarrow 0\right)$. For each $x \in K$, as $n$ gets close to infinity, it holds that

$$
\begin{aligned}
& \left\|f_{n}(x)-x_{n}-\left(f_{0}(x)-x_{0}\right)\right\| \\
& \quad \leq\left\|f_{n}(x)-f_{0}(x)\right\|+\left\|x_{n}-x_{0}\right\| \longrightarrow 0,
\end{aligned}
$$

and, then, we have $\left(f_{n}-C_{x_{n}}\right) \stackrel{\rho}{\rightarrow}\left(f_{0}-C_{x_{0}}\right)$.

(d) $\phi \circ \psi=I_{M}$, where $I_{M}$ is the identity mapping on $M$. For each $f \in M$, clearly,

$$
\phi \circ \psi(f)=\phi\left(f+C_{\bar{x}}, \bar{x}\right)=f+C_{\bar{x}}-C_{\bar{x}}=f,
$$

where $\bar{x}$ is the unique element of $V(f+I)$. (e) $\psi \circ \phi=I_{N}$, where $I_{N}$ is the identity mapping on $N$. For each $\left(f, x^{*}\right) \in N$,

$$
\psi \circ \phi\left(f, x^{*}\right)=\psi\left(f-C_{x^{*}}\right)=\left(f-C_{x^{*}}+C_{\bar{x}}, \bar{x}\right),
$$

where $\bar{x}$ satisfies that $V\left(f-C_{x^{*}}+I\right)=\{\bar{x}\}$. From step (a), we have

$$
(y-\bar{x})^{T}\left(f(\bar{x})-x^{*}+\bar{x}\right) \geq 0, \quad \forall y \in K
$$

Consequently, (11) holds for $y=x^{*}$; that is,

$$
\left(x^{*}-\bar{x}\right)^{T}\left(f(\bar{x})-x^{*}+\bar{x}\right) \geq 0 .
$$

Since $\left(f, x^{*}\right) \in N$, we have $\left(y-x^{*}\right)^{T} f\left(x^{*}\right) \geq 0$, for all $y \in K$. It follows that

$$
\left(\bar{x}-x^{*}\right)^{T} f\left(x^{*}\right) \geq 0 .
$$

Add (12) and (13); then, we obtain that

$$
\left(x^{*}-\bar{x}\right)^{T}\left(-f\left(x^{*}\right)+f(\bar{x})-x^{*}+\bar{x}\right) \geq 0 ;
$$

that is,

$$
\left(x^{*}-\bar{x}\right)^{T}\left(f(\bar{x})+\bar{x}-\left(f\left(x^{*}\right)+x^{*}\right)\right) \geq 0 .
$$

Since $f+I$ is monotone, we have

$$
\left(x^{*}-\bar{x}\right)^{T}\left(f\left(x^{*}\right)+x^{*}-(f(\bar{x})+\bar{x})\right) \geq 0 .
$$

Thus, (15) and (16) imply that

$$
\left(x^{*}-\bar{x}\right)^{T}\left(f\left(x^{*}\right)+x^{*}-(f(\bar{x})+\bar{x})\right)=0 .
$$

Noting that $f+I$ is also strictly monotone, (17) can result in the fact that $x^{*}=\bar{x}$. Therefore, $\psi \circ \phi\left(f, x^{*}\right)=\left(f, x^{*}\right)$.

From the closedness of $N$, the continuity of $\psi$ is guaranteed in Theorem 2. Conversely, $N$ being closed can be induced by Theorem 2 .

Corollary 3. Theorem 2 implies that $N$ is closed.

Proof. Since $(M, \rho)$ is closed, by the continuity of $\psi$ in Theorem 2, we have that $\psi(M)$ is closed. Additionally, Theorem 2 means that $\psi(M)=N$; then, $N$ is closed.

From Theorem 2 or Corollary 3, $N$ is closed, noting that $V(f)$ is closed for each $f \in M$, which results in the upper semicontinuity of the set-valued mapping $V: M \rightarrow 2^{K}$. This directly leads to the following stability result of the solution set $V(f)$ for each $f \in M$.

Corollary 4. Let $\varepsilon>0, f \in M$. For each $\varepsilon$ neighborhood $O_{\varepsilon}$ of $V(f)$ in $K$, there exists a $\delta$ neighborhood $U_{\delta}$ of $f$ in $M$ such that $V\left(f^{\prime}\right) \subset O_{\varepsilon}$, for all $f^{\prime} \in U_{\delta}$. 
Remark 5. As shown in Theorem 2 or Corollary 3, $V$ is upper semicontinuous on $M$. Then, for any $\varepsilon_{n}>0, n=1,2, \ldots$, and $f_{0}+\varepsilon_{n} I \in M$ with a unique solution $x_{n} \in V\left(f_{0}+\varepsilon_{n} I\right)$, we know that $f_{0}+\varepsilon_{n} I \rightarrow f_{0}$. For any $\delta$ open neighborhood $B_{\delta} \supset V\left(f_{0}\right)$, there is a number $m$ such that $x_{n} \in B_{\delta}, n>m$. Then, by the arbitrariness of $\delta$ with $\delta>0$, we can assert that there exists a convergent subsequence $\left\{x_{n_{k}}\right\}$ of $\left\{x_{n}\right\}$ such that $x_{n_{k}} \rightarrow$ $x_{0} \in V\left(f_{0}\right)=\overline{V\left(f_{0}\right)}$. This is like the Tikhonov regularization method for the ill-posed variational inequalities; see [3].

Remark 6. Let $\varepsilon>0$. In Theorem 2, for each $(f, x) \in N$, let $\phi_{\varepsilon}(f, x)=f-\varepsilon C_{x}$ and, for each $f \in M$, let $\psi_{\varepsilon}(f)=$ $\left(f+\varepsilon C_{x}, x\right)$ with $x \in V(f+\varepsilon I)$ for the strongly monotone mapping $f+\varepsilon I$. Then, $\phi_{\varepsilon}$ and $\psi_{\varepsilon}$ are also bijections between $N$ and $M$.

From Theorem 2, in a finite dimensional case, the homeomorphism is shown between two spaces in relation to the variational problem (1). This facilitates the generalization of this property into Hilbert spaces.

In the following part, let $K$ be a compact convex subset in a Hilbert space $(X,\langle\cdot, \cdot\rangle)$, where $\langle\cdot, \cdot\rangle$ denotes the inner product on $X$. We denote by $(X,\|\cdot\|)$ the norm space induced by the inner product on $X$. Let $X^{*}$ be the dual space of $X$ which consists of all bounded linear functions on $X$ equipped with norm topology. Then, for each $T \in X^{*}$, the norm of $T$ can be written as $\|T\|=\sup _{\|y\|=1, y \in X}|T(y)|$. And it is known that $X^{*}$ is a Banach space using this topology.

A mapping $S$ from $K$ to $X^{*}$ is said to be monotone if $(S(u)-S(v), u-v) \geq 0$, for all $u, v \in K$, where $(\cdot, \cdot)$ is the pairing of $X^{*}$ and $X ; S$ is called strictly monotone if $S$ is monotone, and $(S(u)-S(v), u-v)=0$ implies $u=v$.

Let $T: K \rightarrow X^{*}$ be continuous; then, by Theorem 3 in [6], there is $u$ in $K$ solving the variational inequality problem

$$
(T(u), v-u) \geq 0, \quad \forall v \in K
$$

This kind of variational inequality problem was introduced by Browder (see [6]). Denote by $M^{\prime}$ the set of all $T: K \rightarrow$ $X^{*}$ (monotone and continuous on $K$ ) and by $V^{\prime}(T)$ the set of all solutions of the problem (18) for each $T \in M^{\prime}$. Then, we define a set-valued mapping $V^{\prime}$ from $M^{\prime}$ to $K$. For each $T \in M^{\prime}$, we know that if $T$ is strictly monotone, then $V^{\prime}(T)$ is a singleton set. For two $T_{1}, T_{2} \in M^{\prime}$, we measure the metric between them as

$$
\begin{aligned}
\rho^{\prime}\left(T_{1}, T_{2}\right) & =\sup _{x \in K}\left\|T_{1}(x)-T_{2}(x)\right\| \\
& =\sup _{x \in K} \sup _{\|y\|=1, y \in X}\left|\left(T_{1}(x), y\right)-\left(T_{2}(x), y\right)\right| .
\end{aligned}
$$

Denote by $N^{\prime}$ the graph of the set-valued mapping $V^{\prime}$; that is,

$$
N^{\prime}=\left\{(T, x) \in M^{\prime} \times K \mid x \in V^{\prime}(T)\right\} .
$$

To construct a bijection between $M^{\prime}$ and $N^{\prime}$, let $\phi^{\prime}: N^{\prime} \rightarrow$ $M^{\prime}$ such that, for each $(T, u) \in N^{\prime}, \phi^{\prime}(T, u)=T-l_{u}$, where $l_{u}: K \rightarrow X^{*}$ is a constant mapping such that $l_{u}(x)=\langle u, \cdot\rangle$, for all $x \in K$. For each $T \in M^{\prime}$, let $R_{T}: K \rightarrow X^{*}$ such that, for each $x \in K$,

$$
\left(R_{T}(x), z\right)=(T(x), z)+\langle x, z\rangle, \quad \forall z \in X
$$

Easily, we can check that $R_{T}$ is strictly monotone. Let $\psi^{\prime}(T)=$ $\left(T+l_{u}, u\right)$ for each $T \in M^{\prime}$, where $u$ satisfies that $V^{\prime}\left(R_{T}\right)=\{u\}$ and $R_{T}$ is defined by (21).

\section{Theorem 7. The spaces $M^{\prime}$ and $N^{\prime}$ are homeomorphic.}

Proof. We will follow the following five steps to complete the proof.

(a) $\psi^{\prime}$ maps $M^{\prime}$ onto $N^{\prime}$. For each $T \in M^{\prime}$, let $u \in V^{\prime}\left(R_{T}\right)$; we need to show that $u \in V^{\prime}\left(T+l_{u}\right)$. Since $u \in V^{\prime}\left(R_{T}\right)$, we have $\left(R_{T}(u), v-u\right) \geq 0$, for all $v \in K$. Hence,

$$
\begin{aligned}
& (T(u), v-u)+\langle u, v-u\rangle \\
& \quad=\left(T(u)+l_{u}(u), v-u\right) \geq 0, \quad \forall v \in K
\end{aligned}
$$

that is, $u \in V^{\prime}\left(T+l_{u}\right)$.

(b) $\phi^{\prime} \circ \psi^{\prime}=I_{M^{\prime}}$, where $I_{M^{\prime}}$ is the identity mapping on $M^{\prime}$. For each $T \in M^{\prime}$, by step (a), it holds that

$$
\phi^{\prime} \circ \psi^{\prime}(T)=\phi^{\prime}\left(T+l_{u}, u\right)=T+l_{u}-l_{u}=T .
$$

(c) $\psi^{\prime} \circ \phi^{\prime}=I_{N^{\prime}}$, where $I_{N^{\prime}}$ is the identity mapping on $N^{\prime}$. For each $\left(T, u^{*}\right) \in N^{\prime}$,

$$
\psi^{\prime} \circ \phi^{\prime}\left(T, u^{*}\right)=\psi^{\prime}\left(T-l_{u^{*}}\right)=\left(T-l_{u^{*}}+l_{\bar{u}}, \bar{u}\right),
$$

where $\bar{u} \in V^{\prime}\left(R_{T-l_{u^{*}}}\right)$; then, for each $x \in K$,

$$
\left(R_{T-l_{u^{*}}}(x), z\right)=\left(T(x)-l_{u^{*}}(x), z\right)+\langle x, z\rangle, \quad \forall z \in X
$$

On the one hand, by step (a), $\bar{u} \in V^{\prime}\left(T-l_{u^{*}}+l_{\bar{u}}\right)$. Hence,

$$
\left(T(\bar{u})-l_{u^{*}}(\bar{u})+l_{\bar{u}}(\bar{u}), v-\bar{u}\right) \geq 0, \quad \forall v \in K .
$$

Taking a special point $v=u^{*}$ in (26), we can obtain that

$$
\left(T(\bar{u})-l_{u^{*}}(\bar{u})+l_{\bar{u}}(\bar{u}), u^{*}-\bar{u}\right) \geq 0 .
$$

Then,

$$
\left(T(\bar{u})+l_{\bar{u}-u^{*}}(\bar{u}), u^{*}-\bar{u}\right) \geq 0 .
$$

On the other hand, since $u^{*} \in V^{\prime}(T)$, we have that $\left(T\left(u^{*}\right), \bar{u}-\right.$ $\left.u^{*}\right) \geq 0$; hence,

$$
\left(T\left(u^{*}\right), \bar{u}-u^{*}\right)+\left\langle\bar{u}-u^{*}, \bar{u}-u^{*}\right\rangle \geq 0 .
$$

Inequality (29) implies that

$$
\left(T\left(u^{*}\right)+l_{\bar{u}-u^{*}}\left(u^{*}\right), \bar{u}-u^{*}\right) \geq 0 .
$$


Add (28) and (30); it follows that

$$
\left(T(\bar{u})+l_{\bar{u}-u^{*}}(\bar{u})-\left(T\left(u^{*}\right)+l_{\bar{u}-u^{*}}\left(u^{*}\right)\right), u^{*}-\bar{u}\right) \geq 0 .
$$

Noting that $T+l_{\bar{u}-u^{*}}$ is monotone, it holds that

$$
\left(T(\bar{u})+l_{\bar{u}-u^{*}}(\bar{u})-\left(T\left(u^{*}\right)+l_{\bar{u}-u^{*}}\left(u^{*}\right)\right), \bar{u}-u^{*}\right) \geq 0 .
$$

Therefore, by (31) and (32), we have

$$
\left(T(\bar{u})+l_{\bar{u}-u^{*}}(\bar{u})-\left(T\left(u^{*}\right)+l_{\bar{u}-u^{*}}\left(u^{*}\right)\right), \bar{u}-u^{*}\right)=0 .
$$

From the strict monotonicity of $T+l_{\bar{u}-u^{*}}$, we can obtain that $\bar{u}=u^{*}$.

(d) $\phi^{\prime}$ is continuous on $N^{\prime}$. Let $\left(T_{n}, u_{n}\right) \in N^{\prime}, n=1,2, \ldots$, and $\left(T_{n}, u_{n}\right) \rightarrow\left(T_{0}, u_{0}\right) \in N^{\prime}\left(T_{n} \stackrel{\rho^{\prime}}{\rightarrow} T_{0}\right.$ and $\| u_{n}-$ $\left.u_{0} \| \rightarrow 0\right)$. For any $x \in K$, since

$$
\begin{aligned}
& \left\|\left(T_{n}-l_{u_{n}}\right)(x)-\left(T_{0}-l_{u_{0}}\right)(x)\right\| \\
& \quad=\left\|T_{n}(x)-l_{u_{n}}(x)-T_{0}(x)+l_{u_{0}}(x)\right\| \\
& \quad \leq\left\|T_{n}(x)-T_{0}(x)\right\|+\left\|l_{u_{n}}(x)-l_{u_{0}}(x)\right\| \\
& \quad \leq \rho^{\prime}\left(T_{n}, T_{0}\right)+\sup _{\|y\|=1, y \in X}\left|\left\langle u_{n}, y\right\rangle-\left\langle u_{0}, y\right\rangle\right| \rightarrow 0,
\end{aligned}
$$

we have $\left(T_{n}-l_{u_{n}}\right) \stackrel{\rho^{\prime}}{\rightarrow}\left(T_{0}-l_{u_{0}}\right)$. That is, $\phi^{\prime}\left(T_{n}, u_{n}\right) \rightarrow$ $\phi^{\prime}\left(T_{0}, u_{0}\right)$.

(e) $\psi^{\prime}$ is continuous on $M^{\prime}$. Let $T_{n} \in M^{\prime}, n=1,2, \ldots$, and $T_{0} \in M^{\prime}$ with $T_{n} \stackrel{\rho^{\prime}}{\rightarrow} T_{0}, \psi^{\prime}\left(T_{n}\right)=\left(T_{n}+l_{u_{n}}, u_{n}\right)$, and $\psi^{\prime}\left(T_{0}\right)=\left(T_{0}+l_{u_{0}}, u_{0}\right)$. It is sufficient to show that $\left(T_{n}+l_{u_{n}}\right) \stackrel{\rho^{\prime}}{\rightarrow}\left(T_{0}+l_{u_{0}}\right)$ and $\left\|u_{n}-u_{0}\right\| \rightarrow 0$. In order to achieve this, we give a proof of upper semicontinuity of $V^{\prime}$ by the closedness of $N^{\prime}$.

Let $\left\{\left(S_{n}, t_{n}\right)\right\}_{n=1}^{\infty} \subset N^{\prime}$ with $S_{n} \stackrel{\rho^{\prime}}{\rightarrow} S_{0}$ and $\left\|t_{n}-t_{0}\right\| \rightarrow 0$. Then, $t_{n} \in V^{\prime}\left(S_{n}\right)$. That is,

$$
\left(S_{n}\left(t_{n}\right), v-t_{n}\right) \geq 0, \quad \forall v \in K .
$$

By way of contradiction, assume that there exists $w \in K$ such that

$$
\left(S_{0}\left(t_{0}\right), w-t_{0}\right)<0 .
$$

Let $y_{w}=\left(w-t_{0}\right) /\left\|w-t_{0}\right\|, y_{w}^{n}=\left(w-t_{n}\right) /\left\|w-t_{n}\right\|$; then, $\left\|y_{w}^{n}-y_{w}\right\| \rightarrow 0$.

Since $S_{n}$ is continuous on $K, S_{n} \stackrel{\rho^{\prime}}{\rightarrow} S_{0}$, and $\left\|t_{n}-t_{0}\right\| \rightarrow 0$, we have

$$
\begin{aligned}
& \left|\left(S_{n}\left(t_{n}\right), y_{w}^{n}\right)-\left(S_{0}\left(t_{0}\right), y_{w}\right)\right| \\
& \leq \mid\left(S_{n}\left(t_{n}\right), y_{w}^{n}\right)-\left(S_{n}\left(t_{0}\right), y_{w}^{n}\right) \\
& \quad+\left(S_{n}\left(t_{0}\right), y_{w}^{n}\right)-\left(S_{0}\left(t_{0}\right), y_{w}^{n}\right) \\
& \quad+\left(S_{0}\left(t_{0}\right), y_{w}^{n}\right)-\left(S_{0}\left(t_{0}\right), y_{w}\right) \mid \rightarrow 0 .
\end{aligned}
$$

Hence, according to (36), there exists a number $m$ such that

$$
\left(S_{n}\left(t_{n}\right), w-t_{n}\right)<0, \quad \forall n>m .
$$

Clearly, there is contradiction between (35) and (38). Therefore, we have

$$
\left(S_{0}\left(t_{0}\right), v-t_{0}\right) \geq 0, \quad \forall v \in K
$$

that is, $t_{0} \in V^{\prime}\left(S_{0}\right)$. Consequently, the space $N^{\prime}$ is closed and $V^{\prime}$ is upper semicontinuous on $M^{\prime}$. Similar to step (b) in Theorem 2, the fact that $\left\{u_{n}\right\}=V^{\prime}\left(R_{T_{n}}\right)(n=1,2, \ldots)$ and the upper semicontinuity of $V^{\prime}$ imply that $\left\|u_{n}-u_{0}\right\| \rightarrow 0$. Since $\left\|u_{n}-u_{0}\right\| \rightarrow 0$, similar to step (d), we can obtain that $\left(T_{n}+l_{u_{n}}\right) \stackrel{\rho^{\prime}}{\rightarrow}\left(T_{0}+l_{u_{0}}\right)$. The proof is completed.

\section{Conflict of Interests}

The author declares that there is no conflict of interests regarding the publication of this paper.

\section{Acknowledgments}

The author thanks reviewers for their useful and constructive comments. This project is supported by Guangxi Natural Science Foundation (2012GXNSFBA053013), NNSF (61164020), and Doctoral Research Fund of Guilin University of Technology.

\section{References}

[1] P. Hartman and G. Stampacchia, "On some non-linear elliptic differential-functional equations," Acta Mathematica, vol. 115, no. 1, pp. 271-310, 1966.

[2] J. L. Lions and G. Stampacchia, "Variational inequalities," Communications on Pure and Applied Mathematics, vol. 20, no. 3, pp. 493-519, 1967.

[3] F. Facchinei and J. S. Pang, Finite-Dimensional Variational Inequalities and Complementarity Problem, Springer, New York, NY, USA, 2003.

[4] I. V. Konnov, Equilibrium Models and Variational Inequalities, Elsevier, Amsterdam, The Netherlands, 2007.

[5] D. Kinderlehrer and G. Stampacchia, An Introduction to Variational Inequalities and Their Applications, SIAM, Philadelphia, $\mathrm{Pa}, \mathrm{USA}, 2000$.

[6] F. E. Browder, "A new generalization of the Schauder fixed point theorem," Mathematische Annalen, vol. 174, no. 4, pp. 285-290, 1967. 


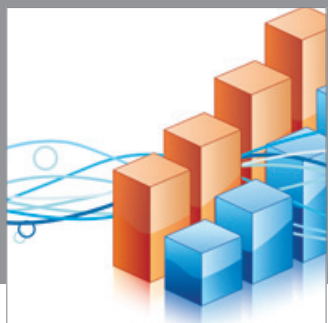

Advances in

Operations Research

mansans

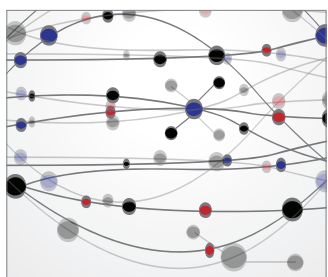

The Scientific World Journal
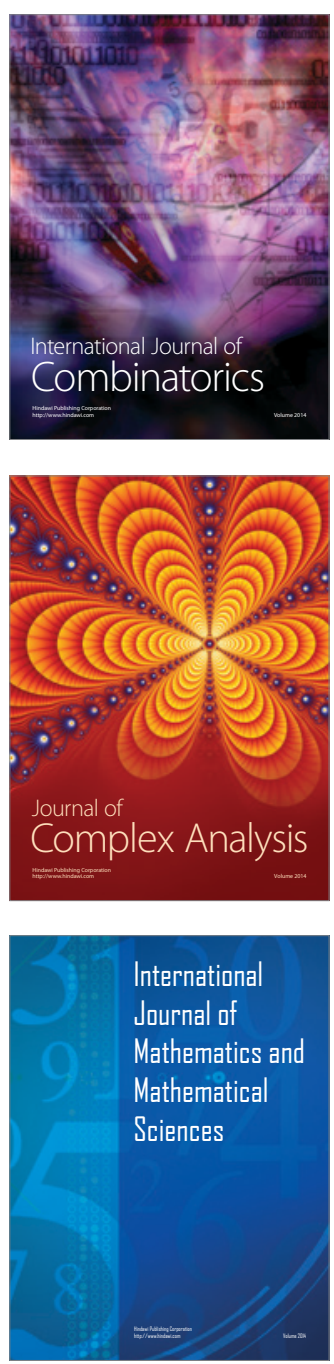
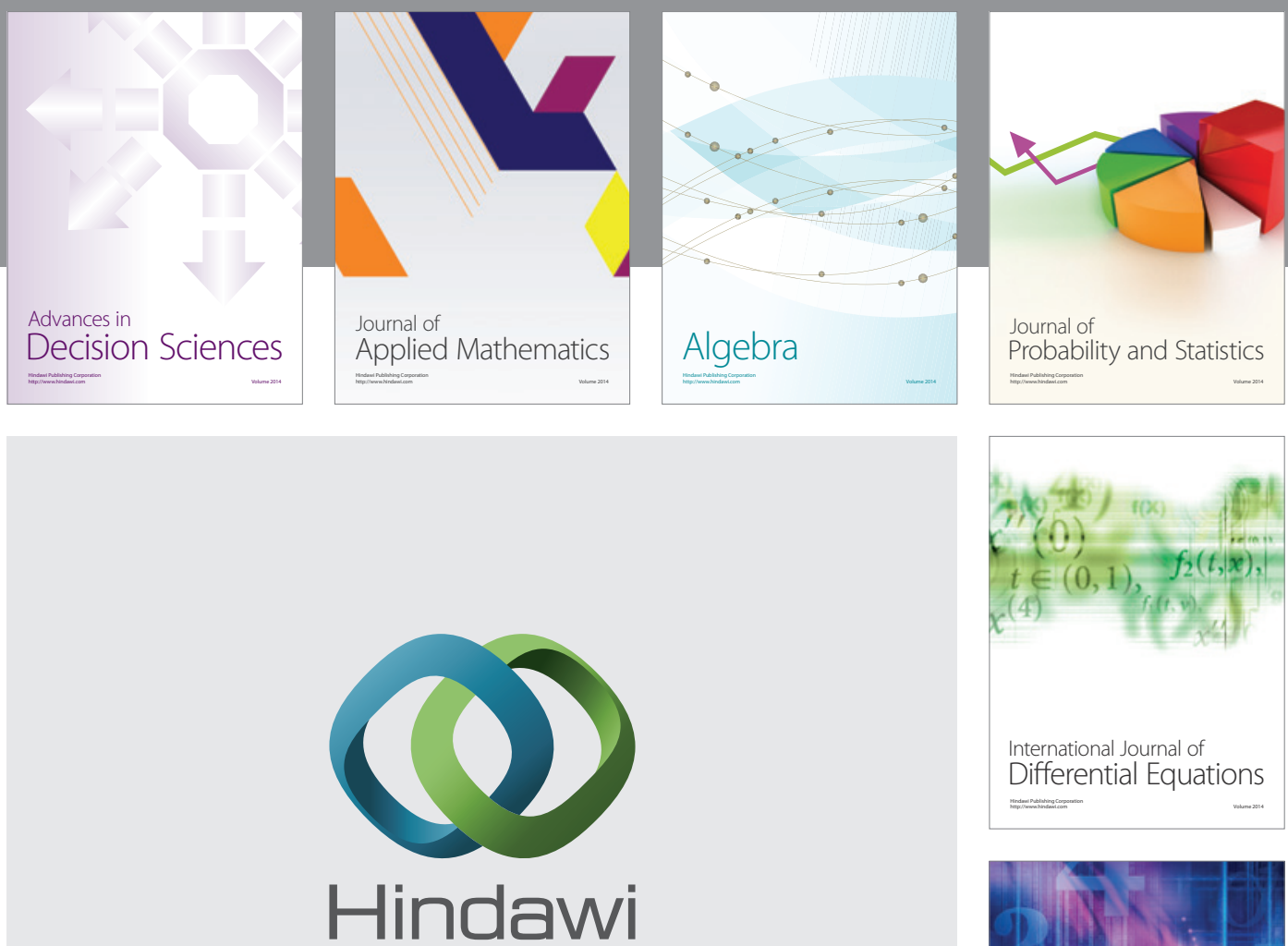

Submit your manuscripts at http://www.hindawi.com
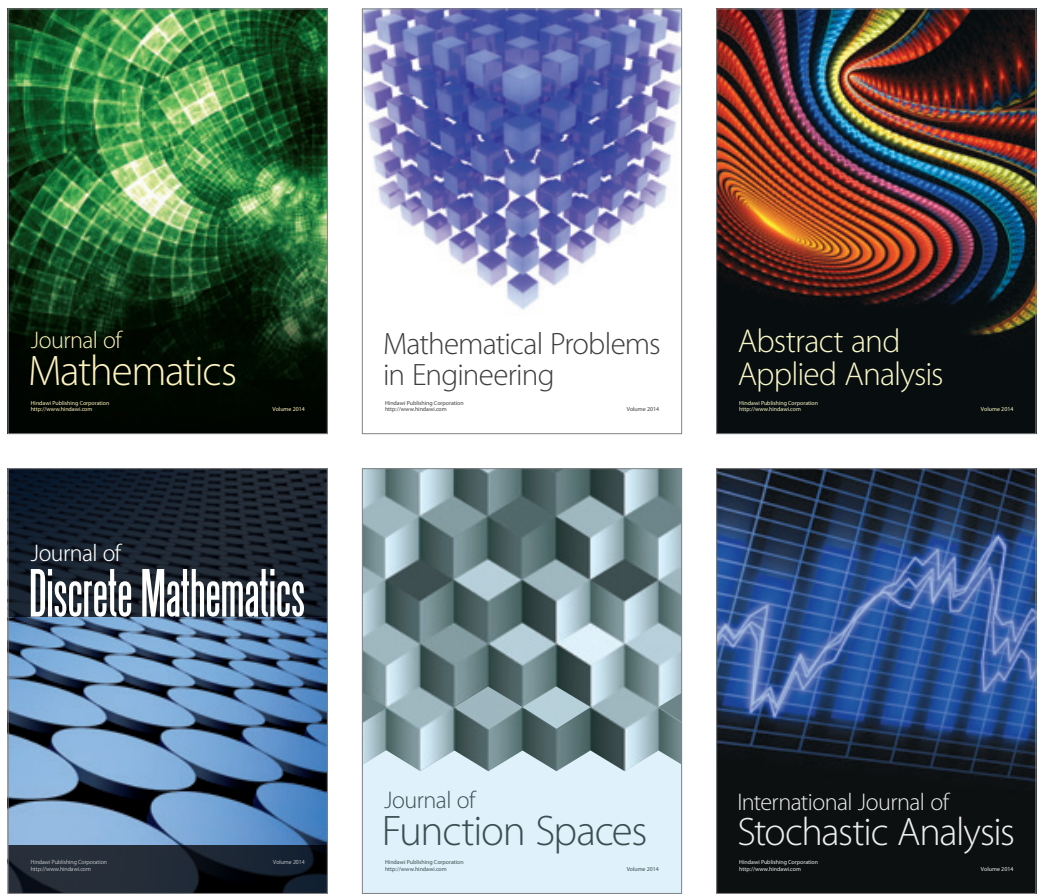

Journal of

Function Spaces

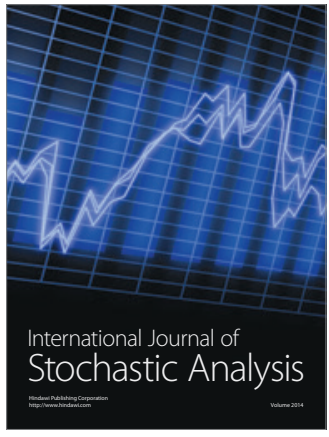

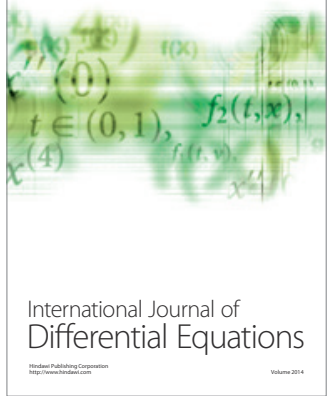
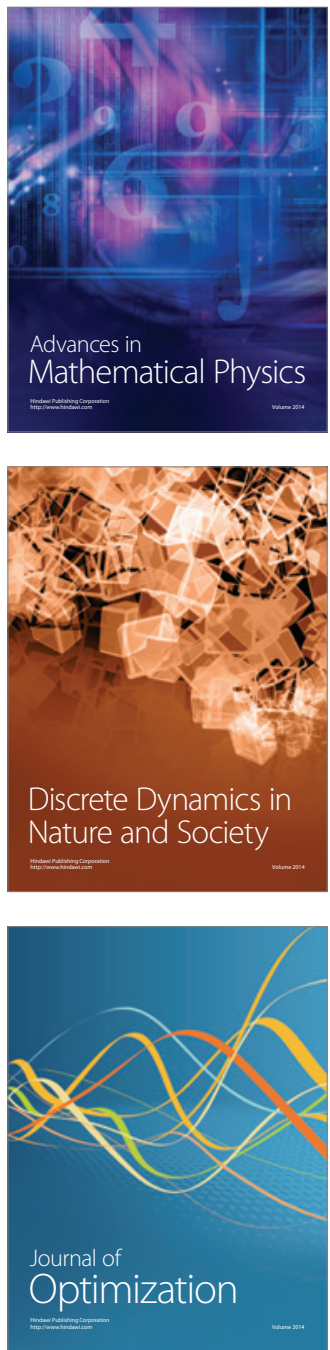